\title{
Engineered recombinant bacterial collagen as an alternative collagen-based biomaterial for tissue engineering
}

\author{
Bo An, David L. Kaplan * and Barbara Brodsky* \\ Department of Biomedical Engineering, Tufts University, Medford, MA, USA \\ *Correspondence: david.kaplan@tufts.edu; barbara.brodsky@tufts.edu \\ Edited by: \\ Carissa M. Soto, Naval Research Laboratory, USA \\ Reviewed by: \\ Lisa Fitzgerald, Naval Research Laboratory, USA
}

Keywords: collagen, triple helix, recombinant expression, biomaterials, tissue engineering

The key structural and signaling roles of collagen in the extracellular matrix (ECM) make it an attractive biomaterial for tissue engineering, but there are limitations in the standardization and purity of natural collagen sources currently available for such applications (Ruggiero and Koch, 2008; Werkmeister and Ramshaw, 2012). Significant effort has been made to produce human collagen in recombinant systems, such as yeast, insect cells and plants (Ruggiero et al., 2000; Myllyharju, 2009). However, the requirement for posttranslational proline hydroxylation has proven to be a significant obstacle in achieving large scale production. Recent findings of collagen-like proteins in bacteria suggest these may represent alternative biosynthetic collagen materials which may complement current sources.

Over the past 10 years, collagen-like proteins have been identified from numerous bacterial genomes database based on the signature (Gly-Xaa-Yaa) $\mathrm{n}$ repeating amino acid sequence characteristic of the collagen triple-helix (Rasmussen et al., 2003; Yu et al., 2014). Some of these collagen-like molecules may function as virulence factors by bacteria to evade the immune system of higher animals or to interact with surface receptors or with other ECM molecules necessary to promote host cell invasion (Humtsoe et al., 2005). More than 100 putative collagen-like proteins have been identified in bacterial genomes, of which eight have been recombinantly expressed in Escherichia coli (see Yu et al., 2014 for review). All eight expressed bacterial collagens were shown to form stable triple-helices with $\mathrm{T}_{\mathrm{m}} \sim 35-39^{\circ} \mathrm{C}$. E. coli and most bacteria lack prolyl hydroxylase, so this high stability is attained in the absence of hydroxyproline (Hyp), a post-translationally modified amino acid known to be critical to the thermal stability of mammalian collagens. Initial interest in bacterial collagen-like proteins focused on their roles in pathogenesis. However, recent work has focused on one specific bacterial collagen protein, designated $\mathrm{Scl} 2$, to demonstrate the utility of recombinant bacterial collagen as a tool for defining collagen sequence/structure/function relationships and for establishing a class of novel collagen-based biomaterials.

The gram positive bacterium Streptococcus pyogenes contains two collagen-like proteins, Scl1 and Scl2, which have been well characterized in terms of structure and functional properties (Lukomski et al., 2001; Xu et al., 2002; Mohs et al., 2007; Caswell et al., 2008). The Scl2 protein includes an N-terminal globular trimerization domain adjacent to a (Gly-Xaa-Yaa) 79 core collagen-like domain. It has been possible to generate constructs in a recombinant $E$. coli system with various sequence modifications of $\mathrm{Scl} 2$ and to establish large scale production methods. Based on recent progress, we suggest that the $\mathrm{Scl} 2$ recombinant bacterial collagen system has advantages compared to recombinant human collagen strategies for large scale production and biomedical applications, and may serve as a prototype for engineering novel collagen-based biomaterials.

\section{STABLE TRIPLE HELICAL PROTEIN WITHOUT HYDROXYPROLINE}

The recombinant $\mathrm{Scl} 2$ protein and its modified variants are able to form a triple helix with stability similar to that of mammalian collagens $\left(\mathrm{T}_{\mathrm{m}} \sim 37^{\circ} \mathrm{C}\right)$ even though there is no Pro hydroxylation. In animal collagens, Pro residues in the $\mathrm{Y}$ positions of the repeating Gly-X$\mathrm{Y}$ sequence are hydroxylated to Hyp, and this hydroxylation is required to stabilize the triple-helix: $\mathrm{T}_{\mathrm{m}}$ of hydroxylated collagen $=37^{\circ} \mathrm{C}$, while $\mathrm{T}_{\mathrm{m}}$ of unhydroxylated collagen $=26^{\circ} \mathrm{C}($ Berg and Prockop, 1973; Jimenez et al., 1973). The bacterial collagen appears to compensate for the absence of Hyp with electrostatic interactions (Mohs et al., 2007). In yeast, plants, and other expression systems for human collagens, the genes for the alpha and beta subunits of human prolyl hydroxylase $(\mathrm{P} 4 \mathrm{H})$ must be introduced to form stable collagen molecules (Ruggiero et al., 2000; Myllyharju, 2009; Xu et al., 2011; Shoseyov et al., 2014). However, $\mathrm{P} 4 \mathrm{H}$ activity and hydroxylation levels are highly dependent on expression conditions, such as the gene copy ratio of collagen to hydroxylase, concentration of cofactors, induction time, and sequence (Chan et al., 2012), so generating optimal hydroxylation to model native human collagen has been challenging in systems with inserted $\mathrm{P} 4 \mathrm{H}$ genes and even in mammalian cell expression systems. The bacterial collagen-like proteins are highly compatible with expression in E. coli, and the lack of requirement for any posttranslational modifications presents the 
potential for producing large quantities of recombinant stable triple-helical proteins for biomaterials applications.

\section{AND MATCH FUNCTIONAL MODULAR UNITS}

In addition to production advantages, the ability to express the collagen-like protein in E. coli allows easy manipulation of the sequence to enhance biomaterial properties. Insertion of known human collagen binding sites within Scl2 presents the possibility of designing collagen-like materials with defined biological properties. The native human collagen sequence contains more than 40 binding sites which interact with other biologically functional molecules, including cell receptors, other ECM proteins and collagenases (MMPs) (Kadler, 1994). Examination of the Scl2 recombinant protein suggests that this particular bacterial collagen-like protein lacks any known biologically active sites, making it a convenient blank slate for the introduction of human collagen ligand binding sites (Caswell et al., 2008; Cosgriff-Hernandez et al., 2010). Various human-derived collagen short amino acid sequences (2-6 triplets) with identified bioactive sites have been inserted into the Scl2 collagen-like domain, including sites for binding integrin $\alpha 2 \beta 1$ (Seo et al., 2010), fibronectin (An et al., 2014), heparin (Peng et al., 2013), and MMPs (Yu et al., 2012). The inserted sequences have conferred the expected biological activities on the Scl2 protein based on in vitro binding, cleavage, and cell culture assays. The Scl2 sequence will apparently fold the short human collagen insert into a triple helix, while the correctly folded human collagen sequence imparts the corresponding biological function to the chimeric protein. The introduction of the integrin and fibronectin binding sites promoted the growth of different types of mammalian cells in vitro, while Scl2 molecules with MMP cleavage sites show enzyme cleavage at the native position. More collagen bioactive sites are being identified each year primarily through synthetic collagen mimetic Toolkit peptides (Farndale et al., 2008), providing an increasing pool of candidates for a recombinant chimeric bacteria-human library with human collagen functions. Such a recombinant library is particularly useful for applying a synthetic biology "plug and play" concept to future biomaterial designs (Figure 1). Only those collagen biological functions that are required for a particular application will be selected and incorporated into a final product, leading to precise tuning for each application.

\section{MOLECULAR AND HIGHER ORDER STRUCTURE}

With recombinant DNA technologies, it has been possible to manipulate the $\mathrm{Scl} 2$ protein, studying individual fragments of the triple-helix (Yu et al., 2011) or duplicating the entire triple-helix portion (Yoshizumi et al., 2009), while maintaining secondary structure and stability. Scl2 is a homotrimer, serving as a model for homotrimeric collagens such as type II collagen in cartilage. To better mimic heterotrimeric collagens such as the dominant type I collagen in bone and tendon or type IV collagen in basement membrane, it may be possible to replace the natural trimerization $\mathrm{V}$ domain with a heterotrimic coiled coil (Nautiyal et al., 1995) or an electrostatic interaction chain selection mechanism (Jalan et al., 2013).

While facile manipulation of the Scl2 protein can optimize molecular and biological properties, successful biomaterial applications will require optimization of material properties as well. The most abundant animal collagens self-associate into characteristic periodic cross-linked fibrils, which provide the mechanical properties for tissues and biomaterials. Thus far, Scl2 exhibits limited ability to form fibrillar structures (Yoshizumi et al., 2009). The Scl2 triple-helix is only about $1 / 5$ of the length of the human fibrillar collagen triple-helix, and it is possible that increasing its length will promote self-association. The E. coli system usually has an upper threshold for the size of the recombinant protein it can produce, but it may be possible to transfer the $\mathrm{Scl} 2$ expression system into yeast or insect cells to further increase its chain length. Crystal structures on small collagen model peptides and studies on recombinant tobacco type I collagen suggest Hyp may be essential for collagen fibril formation (Kramer et al., 2000; Perret et al., 2001). Thus, it may be necessary to introduce $\mathrm{P} 4 \mathrm{H}$ genes into the bacterial system in order to induce fibril formation, even though the Hyp is not necessary to form the stable triplehelix. Alternative approaches to attaining an optimal self-supporting material from Scl2 include chemical modifications such as glutaraldehyde vapor or 1-ethyl-3(3-dimethylaminopropyl)carbodiimide or crosslinking by poly(ethylene glycol) diacrylate to form interchain networks (Cosgriff-Hernandez et al., 2010; Peng et al., 2010). Chemical modifications are simple and effective, but the level of crosslinking maybe difficult to control, especially in large 3D scaffolds, and a high degree of cross-linking may limit accessibility to biologically active sites within the collagen material. Another strategy recently reported involved non-covalent binding of Scl 2 constructs with fibronectin and integrin interaction sites to solid silk protein material, generating porous silk scaffolds with improved support of cell growth (An et al., 2013) (Figure 1).

\section{PURIFICATION, SCALABILITY, AND PROJECTED COST}

Animal extracted collagens are produced in large quantity and are generally inexpensive. However, difficulties in developing standardized preparations of these collagens and in producing minor types of collagens that are free of collagen I and biological contaminants are major limitations. Potential infectious and allergic risks of animal collagen products are also a concern. The recombinant bacterial collagens have attached tags, such as His-tag and Strep II tags to simplify standardized chromatographic purification. A high-throughput batch purification methodology for Scl2 has also been developed (Peng et al., 2014). Native triple helical sequences are resistant to digestion by non-specific enzymes such as trypsin and chymotrypsin, and trypsin treatment of acidified cell lysate resulted in purified triple helical bacterial collagen. Enzymatic digestion during the purification process ensures the final product will be free of non-triple helical contaminants, which is important for quality control for industrial production. Recombinant expression of proteins in E. coli is a mature industrial process with excellent scalability. This has already been demonstrated for $\mathrm{Scl} 2$ production (Peng et al., 2012) with an average yield of $0.2-0.3 \mathrm{~g} / \mathrm{L}$ of purified collagen protein in traditional shaking flask 


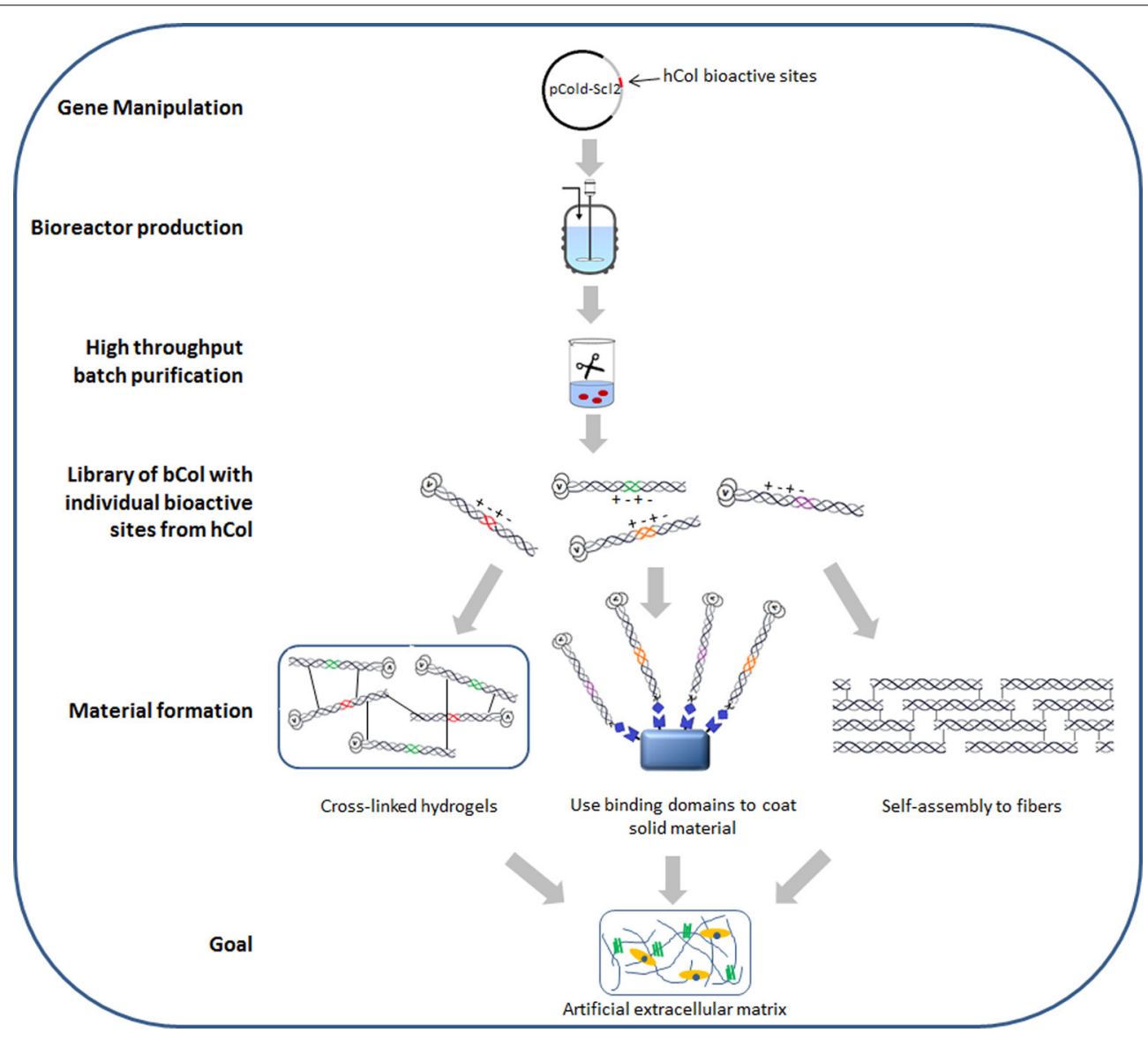

FIGURE 1 | Schematic diagram of recombinant bacterial collagen production method and material fabrication strategies. hCol, human collagen; bCol, recombinant bacterial collagen Scl2.

culture and up to $9.5 \mathrm{~g} / \mathrm{L}$ in high density fed-batch culture. With the ease of generating different Scl2 constructs through molecular cloning, the overall resource cost for producing this highly tunable bacterial collagen material will likely be lower than recombinant collagen obtained from mammalian cell or even transgenic systems.

\section{TOWARD IN VIVO APPLICATIONS}

Translational science remains a less explored area for recombinant collagens. Recombinant human collagens obtained from systems with high scalability, such as Pichia and Nicotiana, have been formulated into hydrogels and explored for their potential as artificial cornea (Merrett et al., 2008) and wound dressings (Shilo et al., 2013), respectively. In terms of bacterial collagen, Scl2 has been shown to be non-immunogenic, non-cytotoxic and non-thrombogenic (Peng et al., 2010).
Recently, poly(ethylene glycol) crosslinked bioactive Scl2 hydrogels have been reinforced with an electrospun polyurethane mesh to achieve suitable biomechanical property for vascular grafts (Browning et al., 2012). The rate of cell migration is tunable through altering protein concentration in the material. Further investigation, especially the degradation and turnover time of Scl2 material in vivo is needed to evaluate its suitability as medical implants.

\section{CRITICAL PROPERTIES FOR A SUCCESSFUL COLLAGEN PRODUCTION STRATEGY}

Figure 1 illustrates the current production method and material fabrication strategies for recombinant bacterial collagen Scl2. Large amounts of bioactive collagen molecules can be produced with simple gene manipulation, large scale production and high throughput purification.
Functional collagen proteins could then be cross-linked into hydrogels or used as coating on other solid materials. Conditions and sequence manipulations which would trigger Scl2 self-assembly into large fibers are currently under investigation. Such fabricated materials could lead to the ultimate goal of designing and developing artificial extracellular matrices, an objective important for tissue engineering as well as biomedical fields. With its high tunability and scalability accompanied by low complexity and cost, we believe the Scl2 recombinant bacterial collagen system has clear advantages which could not only circumvent the difficulties seen for recombinant human collagens, but also open up a brand new pathway for collagen production.

\section{LONG TERM PROSPECTS}

Extracted bovine collagen is likely to remain a staple for biomaterial 
construction due to its low cost and useful material properties, but challenges in standardization and concerns for bioburdens, as well as a desire to modify collagen sequence and function, will lead to continued interest in research and applications with recombinant collagens. As described here for the specific $\mathrm{Scl} 2$ protein from $S$ pyogenes, bacterial collagens represent a biosynthetic ground up approach, where a triple-helical non-animal collagen molecule with no specific bioactivity can be designed to include desired interactions and regulated degradation. Although research has focused on Scl2, the approach could be extended to other collagen-like proteins from bacteria and viruses, which may bring new properties and variations useful for biomaterials. Fundamental studies on $\mathrm{Scl} 2$ and other bacterial collagens will continue to elucidate basic features of collagen, with a focus on chemistry, sequence, biological activity, and selfassembly. The success of the bacterial collagen approach toward biomaterial applications will depend on developing the capacity to generate biomaterials with desirable biomechanical properties useful for in vivo applications.

\section{ACKNOWLEDGMENT}

The authors thank the support from NIH grant\#EB011620 (to Barbara Brodsky and David L. Kaplan) for this work.

\section{REFERENCES}

An, B., Abbonante, V., Yigit, S., Balduini, A., Kaplan, D. L., and Brodsky, B. (2014). Definition of the native and denatured Type II collagen binding site for fibronectin using a recombinant collagen system. J. Biol. Chem. 289, 4941-4951. doi: 10.1074/jbc.M113.530808

An, B., DesRochers, T. M., Qin, G., Xia, X., Thiagarajan, G., Brodsky, B., et al. (2013). The influence of specific binding of collagen, Äisilk chimeras to silk biomaterials on hMSC behavior. Biomaterials 34, 402-412. doi: 10.1016/j.biomaterials.2012.09.085

Berg, R. A., and Prockop, D. J. (1973). The thermal transition of a non-hydroxylated form of collagen. Evidence for a role for hydroxyproline in stabilizing the triple-helix of collagen. Biochem. Biophys. Res. Commun. 52, 115-120. doi: 10.1016/0006291X(73)90961-3

Browning, M. B., Dempsey, D., Guiza, V., Becerra, S., Rivera, J., Russell, B., et al. (2012). Multilayer vascular grafts based on collagen-mimetic proteins. Acta. Biomater. 8, 1010-1021. doi: 10.1016/j.actbio.2011.11.015

Caswell, C. C., Barczyk, M., Keene, D. R., Lukomska, E., Gullberg, D. E., and Lukomski, S. (2008). Identification of the first prokaryotic collagen sequence motif that mediates binding to human collagen receptors, integrins alpha2beta1 and alpha11beta1. J. Biol. Chem. 283, 36168-36175. doi: 10.1074/jbc.M806865200

Chan, S. W., Greaves, J., Da Silva, N. A., and Wang, S. W. (2012). Assaying proline hydroxylation in recombinant collagen variants by liquid chromatography-mass spectrometry. BMC Biotechnol. 12:51. doi: 10.1186/1472-6750-12-51

Cosgriff-Hernandez, E., Hahn, M. S., Russell, B., Wilems, T., Munoz-Pinto, D., Browning, M. B., et al. (2010). Bioactive hydrogels based on designer collagens. Acta. Biomater. 6, 3969-3977. doi: 10.1016/j.actbio.2010. 05.002

Farndale, R. W., Lisman, T., Bihan, D., Hamaia, S., Smerling, C. S., Pugh, N., et al. (2008). Cell-collagen interactions: the use of peptide Toolkits to investigate collagen-receptor interactions. Biochem. Soc. Trans. 36(Pt 2), 241-250. doi: 10.1042/BST0360241

Humtsoe, J. O., Kim, J. K., Xu, Y., Keene, D. R., Hook, M., Lukomski, S., et al. (2005). A streptococcal collagen-like protein interacts with the alpha2beta1 integrin and induces intracellular signaling. J. Biol. Chem. 280, 13848-13857. doi: 10.1074/jbc.M410605200

Jalan, A. A., Demeler, B., and Hartgerink, J. D. (2013). Hydroxyproline-free single composition ABC collagen heterotrimer. J. Am. Chem. Soc. 135, 6014-6017. doi: 10.1021/ja402187t

Jimenez, S., Harsch, M., and Rosenbloom, J. (1973). Hydroxyproline stabilizes the triple helix of chick tendon collagen. Biochem. Biophys. Res. Commun. 52, 106-114. doi: 10.1016/0006-291X(73) 90960-1

Kadler, K. (1994). Extracellular matrix. 1: fibrilforming collagens. Protein Profile 1, 519-638.

Kramer, R. Z., Venugopal, M. G., Bella, J., Mayville, P., Brodsky, B., and Berman, H. M. (2000). Staggered molecular packing in crystals of a collagen-like peptide with a single charged pair. J. Mol. Biol. 301, 1191-1205. doi: 10.1006/jmbi.2000.4017

Lukomski, S., Nakashima, K., Abdi, I., Cipriano, V. J., Shelvin, B. J., Graviss, E. A. (2001). Identification and characterization of a second extracellular collagen-like protein made by group A Streptococcus: control of production at the level of translation. Infect. Immun. 69, 1729-1738. doi: 10.1128/IAI.69.3.1729-1738.2001

Merrett, K., Fagerholm, P., McLaughlin, C. R., Dravida, S., Lagali, N., Shinozaki, N., et al. (2008). Tissue-engineered recombinant human collagenbased corneal substitutes for implantation: performance of type I versus type III collagen. Invest. Ophthalmol. Vis. Sci. 49, 3887-3894. doi: 10.1167/iovs.07-1348

Mohs, A., Silva, T., Yoshida, T., Amin, R., Lukomski, S., Inouye, M. et al. (2007). Mechanism of stabilization of a bacterial collagen triple helix in the absence of hydroxyproline. J. Biol. Chem. 282, 29757-29765. doi: 10.1074/jbc.M703 991200

Myllyharju, J. (2009). Recombinant collagen trimers from insect cells and yeast. Methods Mol. Biol. 522, 51-62. doi: 10.1007/978-1-59745413-1_3

Nautiyal, S., Woolfson, D. N., King, D. S., and Alber, T. (1995). A designed heterotrimeric coiled coil. Biochemistry 34, 11645-11651. doi: 10.1021/bi00037a001

Peng, Y. Y., Howell, L., Stoichevska, V., Werkmeister, J. A., Dumsday, G. J., and Ramshaw, J. A. (2012). Towards scalable production of a collagen-like protein from Streptococcus pyogenes for biomedical applications. Microb. Cell Fact. 11, 146. doi: 10.1186/1475-2859-11-146

Peng, Y. Y., Stoichevska, V., Madsen, S., Howell, L., Dumsday, G. J., Werkmeister, J. A., et al. (2014). A simple cost-effective methodology for large-scale purification of recombinant non-animal collagens. Appl. Microbiol. Biotechnol. 98, 1807-1815. doi: 10.1007/s00253-013-5475-8

Peng, Y. Y., Stoichevska, V., Schacht, K., Werkmeister, J. A., and Ramshaw, J. A. (2013). Engineering multiple biological functional motifs into a blank collagen-like protein template from Streptococcus pyogenes. J. Biomed. Mater. Res. A. 102, 2189-2196. doi: 10.1002/jbm.a.34898

Peng, Y. Y., Yoshizumi, A., Danon, S. J., Glattauer, V., Prokopenko, O., Mirochnitchenko, O., et al. (2010). A Streptococcus pyogenes derived collagen-like protein as a non-cytotoxic and non-immunogenic cross-linkable biomaterial. Biomaterials 31, 2755-2761. doi: 10.1016/j.biomaterials.2009.12.040

Perret, S., Merle, C., Bernocco, S., Berland, P., Garrone, R., Hulmes, D. J., et al. (2001). Unhydroxylated triple helical collagen I produced in transgenic plants provides new clues on the role of hydroxyproline in collagen folding and fibril formation. J. Biol. Chem. 276, 43693-43698. doi: 10.1074/jbc.M105507200

Rasmussen, M., Jacobsson, M., and Bjorck, L. (2003). Genome-based identification and analysis of collagen-related structural motifs in bacterial and viral proteins. J. Biol. Chem. 278, 32313-32316. doi: 10.1074/jbc.M304709200

Ruggiero, F., Exposito, J. Y., Bournat, P., Gruber, V., Perret, S., Comte, J., et al. (2000). Triple helix assembly and processing of human collagen produced in transgenic tobacco plants. FEBS Lett. 469, 132-136. doi: 10.1016/S0014-5793(00)01259-X

Ruggiero, F., and Koch, M. (2008). Making recombinant extracellular matrix proteins. Methods 45, 75-85. doi: 10.1016/j.ymeth.2008.01.003

Seo, N., Russell, B. H., Rivera, J. J., Liang, X., Xu, X., Afshar-Kharghan, V., et al. (2010). An engineered alphal integrin-binding collagenous sequence. J. Biol. Chem. 285, 31046-31054. doi: 10.1074/jbc.M110.151357

Shilo, S., Roth, S., Amzel, T., Harel-Adar, T., Tamir, E., Grynspan, F., et al. (2013). Cutaneous wound healing after treatment with plant-derived human recombinant collagen flowable gel. Tissue Eng. Part A 19, 1519-1526. doi: 10.1089/ten.tea. 2012.0345

Shoseyov, O., Posen, Y., and Grynspan, F. (2014). Human collagen produced in plants: more than just another molecule. Bioengineered 5, 49-52. doi: 10.4161/bioe. 26002

Werkmeister, J. A., and Ramshaw, J. A. (2012). Recombinant protein scaffolds for tissue engineering. Biomed. Mater. 7:012002. doi: 10.1088/17486041/7/1/012002

Xu, X., Gan, Q., Clough, R. C., Pappu, K. M., Howard, J. A., Baez, J. A., et al. (2011). Hydroxylation of recombinant human collagen type I alpha 1 in 
transgenic maize co-expressed with a recombinant human prolyl 4-hydroxylase. BMC Biotechnol. 11:69. doi: 10.1186/1472-6750-11-69

$\mathrm{Xu}$, Y., Keene, D. R., Bujnicki, J. M., Höök, M., and Lukomski, S. (2002). Streptococcal Scl1 and Scl2 proteins form collagen-like triple helices. J. Biol. Chem. 277, 27312-27318. doi: 10.1074/jbc.M201163200

Yoshizumi, A., Yu, Z., Silva, T., Thiagarajan, G., Ramshaw, J. A., Inouye, M., et al. (2009). Selfassociation of streptococcus pyogenes collagen-like constructs into higher order structures. Protein Sci. 18, 1241-1251. doi: 10.1002/pro.134

Yu, Z., An, B., Ramshaw, J. A., and Brodsky, B. (2014). Bacterial collagen-like proteins that form triplehelical structures. J. Struct. Biol. 186, 451-461. doi: 10.1016/j.jsb.2014.01.003
Yu, Z., Brodsky, B., and Inouye, M. (2011). Dissecting a bacterial collagen domain from Streptococcus pyogenes: sequence and length-dependent variations in triple helix stability and folding. J. Biol. Chem. 286, 18960-18968. doi: 10.1074/jbc.M110. 217422

Yu, Z., Visse, R., Inouye, M., Nagase, H., and Brodsky, B. (2012). Defining requirements for collagenase cleavage in collagen Type III using a bacterial collagen system. J. Biol. Chem. 287, 22988-22997. doi: 10.1074/jbc.M112. 348979

Conflict of Interest Statement: The authors declare that the research was conducted in the absence of any commercial or financial relationships that could be construed as a potential conflict of interest.
Received: 21 March 2014; accepted: 04 June 2014; published online: 23 June 2014.

Citation: An B, Kaplan DL and Brodsky B (2014) Engineered recombinant bacterial collagen as an alternative collagen-based biomaterial for tissue engineering. Front. Chem. 2:40. doi: 10.3389/fchem.2014.00040

This article was submitted to Chemical Biology, a section of the journal Frontiers in Chemistry.

Copyright (c) 2014 An, Kaplan and Brodsky. This is an open-access article distributed under the terms of the Creative Commons Attribution License (CC BY). The use, distribution or reproduction in other forums is permitted, provided the original author(s) or licensor are credited and that the original publication in this journal is cited, in accordance with accepted academic practice. No use, distribution or reproduction is permitted which does not comply with these terms. 